\title{
A IMPORTÂNCIA ESTRATÉGICA DOS RELACIONAMENTOS DE NEGÓCIOS EM REDES DE EMPRESAS: UMA VISÃO BASEADA NO RBV - RESOURCE BASED VIEW
}

\section{Carlos Alberto Alves}

Mestre em Administração pela Universidade Paulista - UNIP

Professor de Administração da Universidade Nove de Julho - UNINOVE - FGV

E-mail: calves@uninove.br (Brasil)

\section{Nadia Kassouf Pizzinatto}

Doutora em Administração pela Fundação Getulio Vargas - FGV/SP

Professor do Programa de Mestrado e Doutorado em Administração da Universidade Nove de Julho - UNINOVE

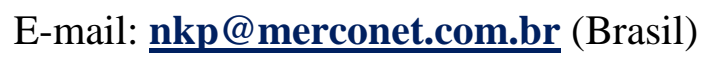

\section{Marcelo Neves Gonçalves}

Mestre em Administração pela Universidade Presbiteriana Mackenzie - MACKENZIE

Professor de Administração e Coordenador da Universidade Nove de Julho - UNINOVE E-mail: marceloneves@uninove.br (Brasil)

\section{RESUMO}

Este trabalho visa discutir a importância dos relacionamentos estratégicos de negócios de forma cooperativa, pois se têm tornado um importante antecedente de sucesso nos negócios. Estudos mostram que alianças e parcerias possuem um papel relevante na moldagem das vantagens competitivas baseadas em recursos da empresa. Para a concretização dos objetivos propostos neste estudo, iniciou-se uma investigação em duas redes imobiliárias e adotou-se como pilar metodológico a tipologia qualitativa de investigação, por fornecer a melhor adequação ao fenômeno observado. Foram utilizados como instrumento: entrevista semiestruturada, pesquisa bibliográfica e observação não participante. Conclui-se que os relacionamentos se mostraram importantes e estratégicos para empresas que estão atuando em formato de redes. Como consequência direta, o acesso aos parceiros e aos recursos que esses possuem pode ser considerado como o mais estratégico dos pontos analisados. Como limite metodológico, deve ser citado o fato de a pesquisa ser de cunho qualitativo. Os resultados têm pouco campo de generalização se comparados a pesquisas quantitativas. A maior implicação prática que se pode advir desta pesquisa é levar aos gestores que estão participando de uma rede a importância estratégica que os relacionamentos possuem. Os gestores deverão considerar, dentro da formulação das suas estratégias, 0 desenvolvimento e a manutenção de relacionamentos para: ter acesso aos parceiros da rede e aos recursos que esses possuem; e construir uma posição forte dentro da rede de forma a poder influenciar outros parceiros

Palavras-chave: Redes de Empresas; Relacionamento de Negócios; Recursos Estratégicos; RBV.

REMark - Revista Brasileira de Marketing, São Paulo, v. 9, n. 2, p 166-189, mai./ago. 2010. 


\section{INTRODUÇÃO}

A habilidade de criar e manter relacionamentos estratégicos de negócios de forma cooperativa tem se tornado um importante antecedente de sucesso nos negócios. O marketing de relacionamento, que se refere a todas as atividades de marketing voltadas para o estabelecimento, desenvolvimento e manutenção de trocas relacionais, materializa-se, nas palavras de Morgan e Hunt, (1994), em contratos relacionais, parcerias de trabalho, marketing simbiótico, alianças estratégicas, alianças de comarketing, e marketing interno. Dessa forma, pode ser considerado parte do paradigma de rede, o qual reconhece que a competição ocorre de modo crescente entre redes de empresas.

O RBV (Resource Based View) ou Visão Baseada em Recursos postula que as empresas são mais lucrativas, não porque investem em barreiras de entrada para os concorrentes ou porque ofereçam produtos diferenciados, mas sim porque elas possuem um conjunto único de recursos seus ativos, competências e capacitações específicas. A teoria por trás do RBV diz que as empresas podem desenvolver vantagens competitivas sustentáveis somente pela criação de valor de modo que essas vantagens sejam raras e difíceis de serem imitadas pelos concorrentes (Barney, 1991, 1995; Grant, 1991).

Estudos têm mostrado que alianças e parcerias possuem um importante papel na moldagem das vantagens competitivas baseadas em recursos da empresa (Lee, Lee, \& Pennings, 2001; McEvily \& Zaheer, 1999; Saxton, 1997; Stuart, 2000; Stuart, Hoang, \& Hybels, 1999). A noção de recursos da rede foi introduzida por Gulati (1998), que examinou como os recursos que são imersosna rede de alianças da empresa moldam os formatos das decisões da aliança. Compartilhada pela maioria dos pesquisadores das redes de empresas, a argumentação de que os relacionamentos de negócios são um dos recursos estratégicos da empresa, não parece ter sido suficientemente discutida e justificada. $\mathrm{O}$ argumento de que os relacionamentos de negócios são estratégicos na natureza e relevância é suportado pelo que realizam para a empresa foco: (a) o acesso aos outros atores da rede e também aos seus recursos e atividades (Gadde, Huemer, \& Hakansson, 2003); (b) a melhoria da posição do ator na rede; e (c) criação de valor potencial dos relacionamentos de negócios. Esses movimentos podem ser necessários, porém não parecem ser condições suficientes para justificar a natureza e a relevância estratégica dos relacionamentos de negócios.

Este estudo parte da questão de que os relacionamentos entre as empresas são os principais fatores para a formação de uma aliança, e de que esse processo é constituído ao longo do tempo. $\mathrm{O}$

REMark - Revista Brasileira de Marketing, São Paulo, v. 9, n. 2, p 166-189, mai./ago. 2010. 
problema de pesquisa pode ser definido da seguinte forma: Os relacionamentos de negócios possuem relevância estratégica para as empresas que atuam em formato de redes?

O presente artigo está organizado da seguinte forma. Inicia-se com uma explicação da perspectiva baseada em redes e em recursos. Na sequência, comenta-se, a importância estratégica dos relacionamentos de negócios para a empresa. Como as premissas básicas da visão baseada em rede e da visão baseada em recursos são completamente diferentes, suas possíveis incompatibilidades são brevemente discutidas em quatro subseções. E finalmente, sumarizam-se algumas questões que necessitam de um estudo mais aprofundado, na tentativa de estender a análise racional baseada em redes, da relevância estratégica dos relacionamentos de negócios. Foi realizado estudo exploratório em duas redes de pequenas empresas de imobiliárias das cidades de Campinas e Indaiatuba, no Estado de São Paulo, com apoio em dados secundários representados por pesquisa em contratos e outros documentos, além de entrevistas com gestores das redes investigadas.

\section{REFERENCIAL TEÓRICO}

\subsection{A PERSPECTIVA BASEADA EM REDE}

As empresas entram em relacionamentos por vários motivos, quais sejam, acesso a novos canais de distribuição, redução de custo, compartilhamento dos riscos, etc. Alguns dos motivos primários para a formação de relacionamentos de negócios são: melhoria das capacidades (capabilities) e recursos internos, ou da criação, acesso ou exploração dos recursos e capacidades externas; desenvolvimento em conjunto de novos recursos ou capacidades; criação em conjunto de novos conhecimentos; redução das incertezas ambientais; coordenação de atividades dissimilares; aumento na eficiência da empresa; e o exercício da influência e do poder na rede.

Pesquisas em alianças focam nas questões de por que e quando as alianças são formadas (Duyster \& Lemmens, 2003; Kogut \& Zander, 1993). Ou seja, o foco são os fatores exógenos que causam a formação de alianças. Interdependência e complementaridade são endereçadas aqui como as explicações para as empresas formarem laços interorganizacionais (Nohria \& Garcia-Pont, 1991; Pfeffer \& Nowak, 1976). Essa perspectiva de dependência de recursos (Wernerfelt, 1984) coloca que os raros e escassos recursos externos são razões importantes para as organizações se engajarem em acordos de colaboração.

REMark - Revista Brasileira de Marketing, São Paulo, v. 9, n. 2, p 166-189, mai./ago. 2010. 
Do ponto de vista da operação das empresas, existem diversas formas de assegurar que as atividades necessárias sejam realizadas: desenvolver as atividades internamente, adquirir empresas que tenham habilidades e recursos especializados, transacionar via mercado e, finalmente, desenvolver alianças estratégicas com outras empresas. Dependendo dos recursos e habilidades disponíveis internamente, da disponibilidade de capital para aquisição desses recursos e da existência de um mercado ofertante dos produtos e serviços necessários, as empresas podem escolher entre as três primeiras alternativas ou, ainda, optar pelo desenvolvimento de alianças estratégicas. O objetivo, nesse caso, é obter as vantagens do desenvolvimento interno das atividades sem arcar com seus custos, garantindo as condições necessárias que, muitas vezes, não são encontradas nas transações de mercado. Alianças estratégicas entre empresas é um fenômeno corriqueiro. Sua proliferação tem levado a um crescimento nas pesquisas realizadas pelos estudiosos em estratégias e organizações e cujo foco tem sido as causas e consequências dessas parcerias, principalmente em nível de díade (Gulati, 1998).

\subsection{A PERSPECTIVA BASEADA EM RECURSOS}

A teoria por trás do RBV diz que as empresas podem desenvolver vantagens competitivas sustentáveis somente pela criação de valor, de modo que essas vantagens sejam raras e difíceis para os competidores imitarem (Barney, 1991, 1995; Grant, 1991).

Para Fleury e Fleury (2003), a Visão Baseada em Recursos postula que as empresas com pessoas, estruturas e sistemas superiores são mais lucrativas, não porque investem em barreiras de entrada contra os concorrentes ou porque ofereçam produtos diferenciados, mas sim porque elas se apropriam das rendas de recursos específicos da empresa. Outra premissa básica da Visão Baseada em Recursos é de que as empresas diferem de forma fundamental em seus modos de operar porque cada uma delas possuirá um conjunto único de recursos - seus ativos, competências e capacitações específicas.

De acordo com Leask (2004), as empresas elaboram suas estratégias baseadas primordialmente em sua combinação de recursos e as oportunidades de mercado que elas identificam. Assim, a teoria da visão baseada em recursos surgiu com o objetivo de desenvolver ferramentas para analisar a posição da empresa em relação aos recursos por ela utilizados e, a partir dessa análise, propor opções de estratégias a serem seguidas pela empresa (Wernefelt, 1984).

REMark - Revista Brasileira de Marketing, São Paulo, v. 9, n. 2, p 166-189, mai./ago. 2010. 
Essa ideia também foi lapidada, as utilidades da teoria se multiplicaram e após maior desenvolvimento, a teoria dos recursos se tornou base para criação de vantagem competitiva (Barney, 1991; Fahy \& Smithee, 1999; Grant, 1991), geração de valor para a empresa (Conner, 1991) e geração de barreiras a novos entrantes (Barney, 1991; Grant, 1991; Wernerfelt, 1984).

Além disso, o RBV pode ser utilizado como modelo de compreensão da estratégia e pode ser considerado a principal referência existente na literatura nessa função (Barney, Wright, \& Ketchen, 2001). A teoria também contribui para o desenvolvimento de capacidades humanas na empresa (Herremans \& Isaac, 2004) e pode ser grande aliada do marketing estratégico (Fahy \& Smithee, 1999).

Algumas limitações merecem uma colocação especial. Lado, Boyd, Wright e Kroll (2006) afirmam que na estratégia do RBV as variáveis teoricamente mais interessantes são aquelas que são menos mensuráveis e identificáveis. Se um recurso não é observável, logo não pode ser imitado facilmente, e cria a base para uma vantagem competitiva sustentável. Contudo, se um recurso não é observável, também não pode ser medido exatamente e empiricamente verificado.

Priem e Butler (2001) também questionam o valor teórico do RBV, especialmente as idéias de Barney (1991), na qual um recurso deve ser valioso, raro e insubstituível para gerar uma vantagem competitiva e sustentável. Baseadas nas ideias de Popper (1959) citado por Priem e Butler (2001), as proposições do RBV são tautológicas - ou seja, são verdadeiras por definição e não podem ser objetivos de testes empíricos.

Collis (1994) argumenta sobre o perigo de a RBV cair em uma regressão infinita, o que significa que o RBV pode levar a uma busca infinita e inútil pelo último estoque de recursos e capacidades que irão gerar uma vantagem competitiva e sustentável. Kraatz e Zajac (2001) afirmam que recursos podem também ser barreiras para o aprendizado, uma vez que as empresas tendem a explorar um recurso já conhecido em vez de partirem para a busca ou desenvolvimento de um novo recurso. Isso se deve ao fato de que os retornos financeiros na exploração de recursos já conhecidos são mais garantidos do que os provenientes de novos recursos ainda por serem desenvolvidos ou descobertos.

REMark - Revista Brasileira de Marketing, São Paulo, v. 9, n. 2, p 166-189, mai./ago. 2010. 


\subsection{A IMPORTÂNCIA ESTRATÉGICA DOS RELACIONAMENTOS DE NEGÓCIOS PARA A EMPRESA}

Diversos autores, como Dyer e Singh (1998), Gadde et al. (2003), Gulati (1995), Gulati, Nohria e Zahher (2000) e Lavie (2006) argumentam que a importância estratégica dos relacionamentos, como defendido na teoria de redes, necessita de mais reflexões e justificativas. Em outras palavras, a importância estratégica dos relacionamentos aparenta ainda não estar totalmente discutida. A argumentação de que os relacionamentos são um dos mais importantes recursos da empresa é frequentemente afirmada, mas nunca suficientemente explicada ou explorada na literatura. Três grandes razões são apresentadas:

Acesso a outros atores na rede e aos seus recursos e atividades - Defendido por Dyer e Singh (1998) e Gadde et al. (2003), é explicito que os relacionamentos de negócios são recursos estratégicos da empresa por que: (a) fornecem acesso aos recursos externos (complementares) e atividades; (b) consideram a maioria dos gastos e aquisições (conectam a empresa aos seus principais clientes e fornecedores) e (c) conectam o ator ao resto da rede de negócios na qual está participando (instituições financeiras, parceiros, rivais e assim por diante).

Melhoria da posição do ator na rede - Relacionamentos de negócios também são vistos como forma de influenciar a posição da empresa na qual está profundamente imersa. Através dos relacionamentos de negócios, um ator pode: (a) influenciar outros atores, os relacionamentos de negócios e consequentemente estruturas de redes e (b) reestruturar a teia de interdependências no sistema de produção (Gadde et al., 2003).

Potencial criação de valor de relacionamentos de negócios - Empresas frequentemente entram em relacionamentos de negócios com o propósito de criação ou apropriação de valor. Esses valores são ambíguos por natureza, somente são subjetivamente medidos e desse modo difíceis de acessarem objetivamente. Além disso, os valores percebidos dos relacionamentos de negócios até certo ponto no tempo dependem de cada episódio de relacionamentos passados.

Todavia, esses motivos não são suficientes para justificar a relevância estratégica dos relacionamentos de negócios, isto é, esses motivos não implicam que todos os relacionamentos de negócios sejam estratégicos para a empresa. Muitos relacionamentos de negócios são terminados (deliberadamente ou não, sem a menor implicação, ou mesmo nenhuma consequência) para uma ou para ambas as empresas envolvidas.

REMark - Revista Brasileira de Marketing, São Paulo, v. 9, n. 2, p 166-189, mai./ago. 2010. 
Essas três classes de argumentos apontadas acima parecem necessárias, porém não são condições suficientes para a relevância estratégica dos relacionamentos de negócios. Uma sugestão é considerar os relacionamentos de negócios como parte dos recursos da empresa (através das lentes do RBV). Isso não seria um absurdo, ao menos para a comunidade de pesquisadores em RBV como alguns teoristas proeminentes: Barney (1991), Teece, Pisano e Shuen (1997) incluíam os relacionamentos de negócios no conjunto de recursos da empresa. As distinções entre os recursos físicos, humanos e organizacionais da empresa incluem os relacionamentos de negócios na categoria de recursos posteriores (Barney, 1991). Amit e Schoemaker (1993) argumentam que os relacionamentos de negócios podem ser um dos ativos estratégicos da empresa (recursos e capacidades que são difíceis de negociar e imitar, e que conferem uma vantagem competitiva), considerando a sugestão, Teece et al. (1997) sugerem que os relacionamentos de negócios são parte dos ativos de posicionamento da empresa e moldam os processos gerenciais e organizacionais contribuindo para sua vantagem competitiva.

\subsection{INCOMPATIBILIDADE POTENCIAL DA REDE E DA PERSPECTIVA DE BASEADA EM RECURSOS}

O argumento básico é que o RBV pode ser apropriado para a avaliação da importância estratégica dos relacionamentos de negócios. Naturalmente, se reconhece previsivelmente que a $\mathrm{RBV}$ e as redes possuem interesses divergentes. O RBV foca nas explicações diferenciais de desempenho através das empresas, considerando que as redes estão interessadas com as características e evoluções dos sistemas empresariais (a rede intricada de relacionamentos de negócios que complexamente ligam as empresas), embora envolvendo a empresa foco.

Consequentemente, essas perspectivas teóricas diferem na suas unidades principais de análises, que são os recursos da empresa e respectivamente os relacionamentos de negócios da rede.

Entretanto, a perspectiva baseada em rede e a perspectiva baseada em recursos diferem-se fortemente em termos de suas premissas com relação a empresa, ambiente, recursos e estratégias. Ver o Quadro 1 para detalhes de cada premissa.

REMark - Revista Brasileira de Marketing, São Paulo, v. 9, n. 2, p 166-189, mai./ago. 2010. 


\begin{tabular}{|c|c|c|}
\hline QUESTÃO & PERSPECTIVA BASEADA EM REDE & $\begin{array}{c}\text { PERSPECTIVA BASEADA EM } \\
\text { RECURSOS }\end{array}$ \\
\hline Unidade de Análise & Rede & Empresa \\
\hline Empresa & $\begin{array}{l}\text { Ator da rede; } \\
\text { Orientada a transação; } \\
\text { Coleção de recursos; } \\
\text { Estruturada em atividades; } \\
\text { Limites indefinidos e obscuros (mudam } \\
\text { conforme as interações entre os atores). }\end{array}$ & $\begin{array}{l}\text { Entidade independente e isolada; } \\
\text { Orientada a produção; } \\
\text { Conjunto de recursos; } \\
\text { Entidade possuidora de propriedade; } \\
\text { Limites claros e definidos (definidos } \\
\text { pelos direitos de propriedade). }\end{array}$ \\
\hline Ambiente & $\begin{array}{l}\text { Em rede, cooperativo e parcialmente } \\
\text { influenciável. }\end{array}$ & $\begin{array}{l}\text { Atomístico, sem face, hostil e } \\
\text { incontrolável. }\end{array}$ \\
\hline Recursos & $\begin{array}{l}\text { Definido pela propriedade e controle, } \\
\text { acessado através de critérios de } \\
\text { relacionamentos; } \\
\text { Variável (Alterando em propriedade e nas } \\
\text { dimensões de uso); } \\
\text { Heterogeneidade do recurso como ganhos } \\
\text { das operações internas e dos processos de } \\
\text { troca; } \\
\text { Recursos estratégicos podem se estender } \\
\text { além dos limites da empresa; } \\
\text { Complementaridade dos recursos intra e } \\
\text { inter-empresas. }\end{array}$ & $\begin{array}{l}\text { Definidos somente pelos critérios de } \\
\text { controle e posse; } \\
\text { Largamente imutáveis (mudando apenas } \\
\text { nos critérios de propriedade); } \\
\text { Heterogeneidade dos recursos como é } \\
\text { dado; } \\
\text { Recursos estratégicos (alojados somente } \\
\text { dentro da empresa); } \\
\text { Complementaridade de recursos intra- } \\
\text { empresa; } \\
\text { Relação competitiva entre recursos } \\
\text { internos e externos. }\end{array}$ \\
\hline Estratégia & $\begin{array}{l}\text { Explorando a geração de ganhos dos } \\
\text { recursos internos e externos; } \\
\text { Construção ou fortalecimento da posição } \\
\text { na rede; } \\
\text { Estratégias de relações }\end{array}$ & $\begin{array}{l}\text { Organizando recursos internos; } \\
\text { Explorando fatores de mercado ou } \\
\text { imperfeições; } \\
\text { Decidindo sobre o desenvolvimento ou a } \\
\text { organização de recursos; } \\
\text { Estratégia competitiva. }\end{array}$ \\
\hline
\end{tabular}

Quadro 1 - Comparação entre Perspectiva Baseada em Rede e Perspectiva Baseada em Recursos. Fonte: Elaborado pelos autores. 


\subsubsection{A POSSIBILIDADE REAL DO CRUZAMENTO ENTRE A PERSPECTIVA BASEADA EM REDE E A PRESPECTIVA EM RECURSOS}

O raciocínio dentro do RBV é o de que a noção de recursos estratégicos está fortemente relacionada ao conceito de vantagem competitiva (a habilidade de criar, sustentar e renovar ganhos). A noção da vantagem competitiva pode ser compatível com a perspectiva baseada em rede - basicamente, a posição da empresa na rede e seus relacionamentos estratégicos podem ser vistos como concentrando (criando, sustentando e renovando) ganhos correntes e antecipados. Essa noção de vantagem competitiva e posicionamento na rede podem coexistir, com algumas empresas operando simultaneamente em um mercado atomístico e em uma rede emaranhada de relacionamentos de negócios.

$\mathrm{O}$ argumento utilizado é o de que a possibilidade real de cruzamento entre as perspectivas baseadas em recursos e as baseadas em redes pode não ser somente oposta, mas também conceitualmente proveitosa para ambos os campos de estudos. Dessa forma, o RBV pode dominar algumas deficiências, nominalmente suas visões impraticáveis da empresa isolada (consequentemente ignorando recursos externos e contribuições à vantagem competitiva da empresa), resultando em limitações na gestão do desempenho da empresa em rede (Lavie, 2006).

Como colocam Gulati et al. (2000):

o comportamento e a desempenho da firma podem facilmente ser entendido pelo exame dos relacionamentos da rede na qual a firma se encontra imersa. Pela adoção de uma aproximação relacional em vez de uma aproximação atomística, podemos aprofundar nosso entendimento das fontes de diferenças no comportamento e na rentabilidade da firma ( $\mathrm{p}$. 203).

Já a visão em redes pode aprimorar a compreensão da natureza e da relevância estratégica dos relacionamentos de negócios. O RBV pode espalhar uma luz em ambos os relacionamentos de negócios (quais características distinguem relacionamentos estratégicos dos não estratégicos), e as relevâncias estratégicas (quais consequências, em termos de capacidades, advêm do envolvimento da empresa em relacionamento de negócios estratégicos e não estratégicos).

A ponte entre essas duas perspectivas foi obtida por Loasby (1998), que unindo tanto os insights de Penrose como os de Richardson, harmonizou a lógica baseada em recursos e a lógica baseada em redes. Loasby (1998) postula que sendo a empresa um conjunto (direto e indireto) de

REMark - Revista Brasileira de Marketing, São Paulo, v. 9, n. 2, p 166-189, mai./ago. 2010. 
capacidades também se encontra imersa em uma rede de empresas diversificadas com seus próprios conjuntos de capacidades (diretas e indiretas). Loasby, (1998), coloca que:

Se iniciarmos uma análise, não com um ideal de competitividade perfeito e uma ênfase em um contrato bem especificado, mas com uma capacidade direta e limitada, e consequentemente com a necessidade de obter coisas feitas por outros, então devemos reconhecer a possibilidade de construir relacionamentos para gerenciar de forma próxima capacidades complementares e naturalmente as vantagens substanciais em muitas circunstâncias de se fazer isso (p. 15).

\subsection{MODELO TEÓRICO PROPOSTO}

Baseado no trabalho de Alves e Tiergarten (2008) utiliza-se o modelo teórico, no qual a vantagem competitiva aparece como consequência do acesso a outros atores na rede e aos seus recursos e atividades; melhoria da posição do ator na rede; e na potencial criação de valor de relacionamentos de negócios. A lógica representada no modelo é a de que uma empresa pode obter uma vantagem competitiva através dos relacionamentos com outras empresas. O esquema é apresentado na Figura 1.

Figura 1 - Modelo Teórico Sobre a Vantagem Competitiva a Partir dos Relacionamentos da Empresa.

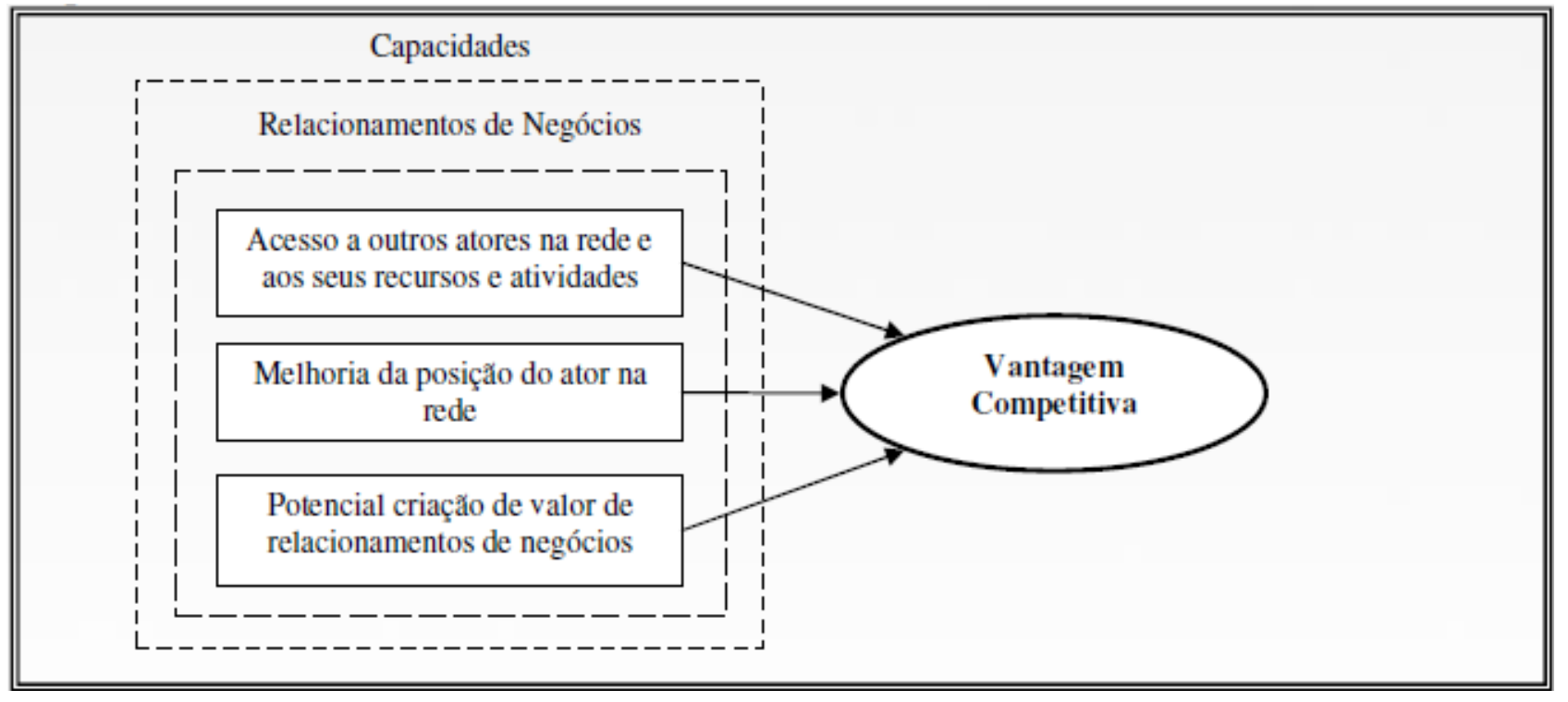

Fonte: Adaptado de Alves e Tiergarten (2008).

REMark - Revista Brasileira de Marketing, São Paulo, v. 9, n. 2, p 166-189, mai./ago. 2010. 


\section{PERSPECTIVA METODOLÓGICA}

Para a concretização dos objetivos propostos neste estudo, adotou-se como pilar metodológico a tipologia qualitativa de investigação, por fornecer a melhor adequação ao fenômeno observado (Patton, 2002; Vieira \& Zouain, 2004). Quanto aos fins, esta pesquisa pode ser considerada exploratória (Gil, 2002).

Como objeto a ser estudado, optou-se por um estudo de caso múltiplo centrado em duas redes de pequenas empresas de imobiliárias das cidades de Campinas e Indaiatuba, no Estado de São Paulo. O estudo de caso é apresentado por Vergara (2003) como metodologia direcionada para o conhecimento em profundidade de uma determinada problemática e tem como maior limitação a impossibilidade de generalização dos resultados para outras empresas. Para Eisenhardt (1989), o estudo de caso é uma estratégia de pesquisa, a qual foca no entendimento da dinâmica, presente dentro de cenários únicos. Porém, apresenta como grande vantagem o fato de os dados levantados representarem de forma mais completa a realidade pesquisada.

Para efeito de levantamento dos dados, utilizou-se da convergência de várias fontes de evidências, sendo selecionadas entrevistas semiestruturadas (gravadas mediante prévia autorização dos entrevistados), análise de documentos e a observação não participante.

\subsection{PROCEDIMENTO DE COLETA}

As unidades de observação pesquisadas foram as pessoas que ocupam cargos classificados como pertencentes à diretoria nas empresas. Os dados foram coletados através de entrevista semiestruturada. A escolha da entrevista como técnica é explicada por possibilitar uma interação maior entre o pesquisador e os pesquisados. Conforme Lüdke e André (1996, p. 33), "na entrevista a relação que se cria é de interação, havendo uma atmosfera de influência recíproca entre quem pergunta e quem responde".

O tratamento dos dados consistiu de uma análise do conteúdo do tipo temática das entrevistas realizadas, das análises de documentos coletados e da observação não participante. $\mathrm{O}$ método de análise de conteúdo é constituído por um conjunto de técnicas (classificação de conceitos, codificação e categorização) que permite estudar as comunicações entre os homens, enfatizando o conteúdo das mensagens. Para Bardin (1977), a análise de conteúdo é uma técnica que visa compreender o sentido de um texto. Para Martins (2006) a análise de conteúdo busca a

REMark - Revista Brasileira de Marketing, São Paulo, v. 9, n. 2, p 166-189, mai./ago. 2010. 
essência da substância de um contexto nos detalhes dos dados e informações disponíveis. O interesse não se restringe à descrição dos conteúdos. Portanto, as entrevistas foram transcritas para a realização da análise de conteúdo das mensagens e, então, realizadas as inferências a partir das informações obtidas. Na medida em que os temas foram aparecendo nos textos, eles foram sendo classificados de acordo com as unidades de análises centrais. A frequência simples foi a medida utilizada. Em síntese, as categorias geradas foram as seguintes: acesso a outros atores na rede e aos seus recursos e atividades; melhoria da posição do ator na rede; e potencial criação de valor baseado nos relacionamentos de negócios.

Para a análise documental, realizaram-se a seleção e os procedimentos de aquisição dos documentos considerados importantes para o tema, ponderando-se os limites de acesso aos documentos referentes às alianças. Os documentos aos quais os pesquisadores tiveram acesso para análise foram: propostas e contratos de serviços; análises de correspondência entre os parceiros da aliança; memorandos internos; além desses dados secundários, utilizou-se uma fonte primária de informação de Estudos Exploratórios, a das pessoas com experiência (Mattar, 1996) por meio do depoimento de alguns parceiros que se prontificaram a responder algumas questões sobre o nível de relacionamento com as empresas em análise. A pesquisa utilizou também a técnica de triangulação de dados, ou seja, a inter-relação entre as informações obtidas dos dados, fontes primárias e secundárias, com o intuito de ampliar a compreensão do estudo em questão e aumentar a fidedignidade e coerência das conclusões.

As entrevistas foram realizadas no período compreendido entre o final de 2008 e durante o ano de 2009; 31 empresas participaram da pesquisa e um total de 38 pessoas foram entrevistadas; os cargos variaram de proprietários a gerentes comerciais, cujas funções previam a responsabilidade de se relacionarem com as empresas que pertenciam à rede e conduzirem negócios. O roteiro de entrevista teve os seguintes tópicos: (1) questões de cunho geral; (2) questões sobre a estrutura da rede; (3) questões sobre acesso a outros atores na rede e aos seus recursos e atividades; (4) questões sobre a posição do ator na rede; (5) questões sobre criação de valor baseado nos relacionamentos de negócios.

As notas das observações de campo também foram analisadas utilizando-se o método de análise de conteúdo.

REMark - Revista Brasileira de Marketing, São Paulo, v. 9, n. 2, p 166-189, mai./ago. 2010. 


\section{RESULTADOS ALCANÇADOS}

Neste item apresentamos e discutimos os resultados alcançados por meio do trabalho de campo. O objetivo é verificar se as afirmativas iniciais são sustentadas ou não pelos dados do campo.

\subsection{APRESENTAÇÃO DAS REDES E SEUS CONTRIBUINTES}

As redes escolhidas como caso para o estudo são do setor imobiliário, as duas redes se encontram respectivamente nas cidades de Indaiatuba e Campinas. São 13 empresas na rede imobiliária de Indaiatuba e 30 na rede imobiliária de Campinas. Do total de 43 empresas apenas dez em Indaiatuba e 21 em Campinas participaram das entrevistas.

As redes imobiliárias de Indaiatuba e Campinas são união e parceria entre empresas do segmento de imobiliárias da região da cidade. A iniciativa tem como principal objetivo reunir um grupo de imobiliárias para comercializar imóveis de maneira conjunta, facilitando a captação, venda e locação. Outro objetivo da rede é disciplinar a conduta comercial, com a implantação de regras de trabalho.

Os meios para atingir essa evolução são o constante trabalho de integração dos participantes (encontros, eventos, reuniões, debates), investimentos regulares em treinamento (cursos, palestras, seminários, workshops) e marketing (criação de marca própria, planejamento estratégico, campanhas publicitárias).

O funcionamento básico da rede para o mercado consiste no conceito de exclusividade. $\mathrm{O}$ cliente assina um termo em uma imobiliária da rede e o imóvel em questão é imediatamente colocado no sistema de informática, integrado por todas as imobiliárias participantes.

Automaticamente está disponibilizado para todos os profissionais trabalharem a venda, ou seja: para vender, comprar ou alugar, basta a pessoa procurar uma imobiliária ou um corretor e terá à disposição o banco de dados unificado de todo o grupo, multiplicando opções de escolha, ganhando agilidade e reduzindo desperdício de tempo.

Importante destacar que a Rede Imobiliária de Indaiatuba tem seu modelo de negócio inspirado na Rede Imobiliária de Campinas, da qual copiou diversas práticas, documentos e exemplos. Por isso, e pela proximidade geográfica, vários resultados encontrados entre as redes são similares. A diferença se dá nos relacionamentos pessoais: esse dado é mais consistente em

REMark - Revista Brasileira de Marketing, São Paulo, v. 9, n. 2, p 166-189, mai./ago. 2010. 
Indaiatuba do que em Campinas, pois é cidade pequena, todos se conhecem, e que cresceu nos últimos anos por causa da expansão econômica da região. A segunda rede, de Campinas, pertence a uma cidade de porte médio para grande, na qual os relacionamentos são muito reduzidos ou inexistentes, se comparado à outra.

\subsection{ACESSO A OUTROS ATORES NA REDE E AOS SEUS RECURSOS E ATIVIDADES}

Feitas as entrevistas nas duas redes, encontrou-se evidências que comprovam que esse constructo possui importância dentro do modelo estratégico das redes de empresas. Negócios conduzidos nas duas redes pelos atores mostraram que a possibilidade de acessar os recursos disponíveis é essencialmente uma vantagem estratégica, já que amplia o número de possibilidades de negócios. No ramo imobiliário existem dois recursos importantes que um corretor necessita possuir, o número de imóveis disponíveis em carteira para vendas e o número de clientes interessados. Esses dois recursos são muito utilizados pelos atores dentro da rede de imobiliárias já que uma empresa acessa a carteira de qualquer outra empresa na rede, multiplicando suas oportunidades negócios.

Os pesquisadores tiveram acesso a três tipos principais de documentos: e-mails, contratos e propostas comerciais; em todos os casos ficou evidente que o acesso aos recursos de outros atores foi um diferencial em termos de negócios para as imobiliárias da rede. Em diversas propostas enviadas a clientes constavam produtos que pertenciam a carteira de outro ator dentro da rede (tanto de Indaiatuba como a de Campinas). Podemos afirmar que o principal motivo para a associação das empresas no formato rede é justamente o acesso aos outros atores e seus recursos. Os dados oriundos dos documentos coletados dentro das duas redes pesquisadas foram conclusivos.

A observação não participante revelou que o acesso a outros atores dentro das duas redes pesquisadas permitia que uma empresa tivesse acesso de forma mais rápida aos recursos da outra empresa, porém nem todas as empresas se utilizavam dessa estratégia da mesma forma. Aquelas mais bem posicionadas eram as que tinham o maior acesso a esses recursos.

REMark - Revista Brasileira de Marketing, São Paulo, v. 9, n. 2, p 166-189, mai./ago. 2010. 


\subsection{A POSIÇÃO DO ATOR NA REDE}

Feitas as entrevistas nas duas redes, encontrou-se evidências que os resultados mostraram que, juntamente com acesso aos outros atores e seus recursos, os atores mais bem posicionados são os que também mais compartilham os recursos disponíveis.

Utilizando o programa de computador UCINET, para análise de redes sociais, e com os resultados obtidos com as entrevistas, foi possível montar um mapa de nós e laços (Erro! Fonte de referência não encontrada.), no qual os pontos marcados com $\mathrm{C}$ referem-se às imobiliárias de Campinas e os pontos marcados com I, às imobiliárias de Indaiatuba. A distribuição e as conexões das empresas representam, nesse caso específico, um mapa com o cruzamento dos recursos que estão disponíveis e que foram acessados pelos diversos atores das duas redes analisadas. Mais conexões, mais recursos disponíveis, resultando em mais negócios para os atores. Foi possível, mapeando os atores, encontrar aqueles com melhores posições dentro da rede, o que comprovou também a afirmativa anterior, pois eram os que melhor acessavam os recursos disponíveis.

Diferentemente das entrevistas, os dados oriundos dos documentos coletados, dentro das duas redes pesquisadas, não mostraram evidências da melhoria da posição dos atores como um constructo válido. Porém quando partimos para a observação não participante, ficou evidente que o constructo possui validade, já que voltam a aparecer os atores mais bem posicionados como os que mais vantagens usufruem dos recursos disponíveis.

Figura 2 - Mapa de nós e conexões das redes imobiliárias de Indaiatuba e Campinas.

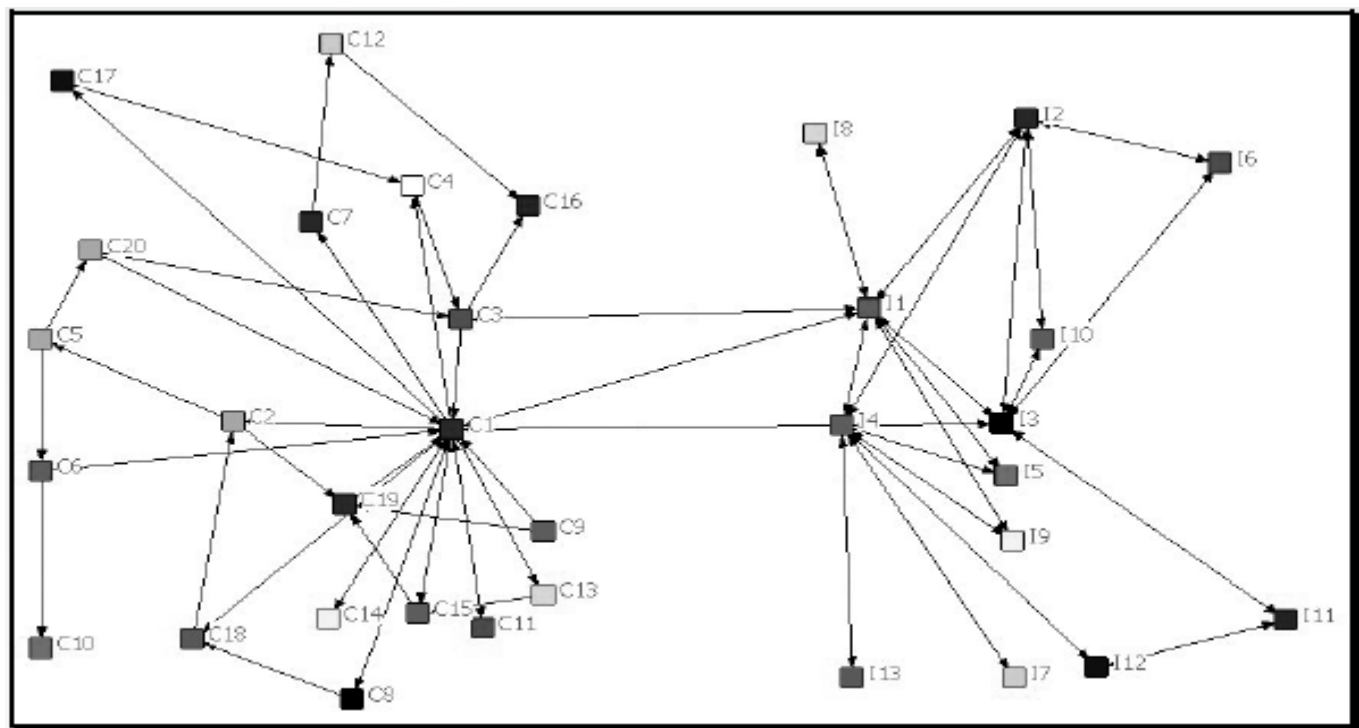

Fonte: Elaborado pelos autores.

REMark - Revista Brasileira de Marketing, São Paulo, v. 9, n. 2, p 166-189, mai./ago. 2010. 


\subsection{POTENCIAL CRIAÇÃO DE VALOR BASEADO NOS RELACIONAMENTOS DE NEGÓCIO}

Nas entrevistas, o constructo potencial criação de valor baseado nos relacionamentos foi o fator motivador para a entrada das empresas nas duas redes pesquisadas. Todas as empresas, sem exceção, tinham essa dimensão muito bem caracterizada como objetivo estratégico, já que criar valor significaria acesso aos recursos dos parceiros.

Nos documentos que os pesquisadores tiveram acesso não foi possível identificar o constructo em análise. E na observação não participante o resultado foi o mesmo.

\subsection{A TRIANGULAÇÃO DOS RESULTADOS}

Triangulando os dados coletados, resultantes das entrevistas semiestruturadas, dos documentos analisados e da observação não participante, foi possível encontrar alguns resultados interessantes.

$\mathrm{O}$ acesso a outros atores na rede e aos seus recursos e atividades aparece nos três instrumentos de coletas de dados, esse constructo possui uma dimensão muito bem estruturada em qualquer uma das empresas que pertencem as redes analisadas. É de entendimento de todas as empresas que acessar os parceiros e seus recursos é um fator relevante e estratégico.

A posição do ator na rede não aparece nos três instrumentos de coletas de dados, mas em apenas um, nas entrevistas. A coleta de documentos e a observação não participante não evidenciaram esse constructo.

Criação de valor baseado nos relacionamentos de negócios também não aparece nos três instrumentos de coletas de dados, aparece somente nas entrevistas. A coleta de documentos e a observação não participante não registram esse constructo.

REMark - Revista Brasileira de Marketing, São Paulo, v. 9, n. 2, p 166-189, mai./ago. 2010. 


\section{CONCLUSÕES E RECOMENDAÇÕES PARA NOVAS PESQUISAS}

Recuperando a questão inicial desta pesquisa: Os relacionamentos de negócios possuem relevância estratégica para as empresas que atuam em formato de redes? Como defendido por Dyer e Singh (1998) e Gadde et al. (2003), conclui-se que os relacionamentos se mostraram importantes e estratégicos para empresas que estão atuando em formato de redes. Como consequência direta, pode-se afirmar que o acesso aos parceiros e aos recursos que esses possuem pode ser considerado como o mais estratégico dos pontos analisados. Na sequência, a melhoria da posição dentro da rede somente é considerada quando vista como um caminho para melhorar o acesso aos parceiros e seus recursos; não foram encontradas evidências que corroborem as afirmativas defendidas por Gadde ET al. (2003). E finalmente, o constructo potencial criação de valor é subjetivo, pois somente foi considerado pelos atores dentro das duas redes pesquisadas como motivo inicial para se associar à rede de empresas. Entendemos que esses dois constructos carecem de mais estudos e pesquisas para que resultados mais robustos possam ser encontrados e considerados (Erro! Fonte de referência não encontrada.).

Outra consideração a ser feita foi a percepção dos pesquisadores com relação às duas redes de empresas. Embora as redes estejam em cidades do interior paulista, foi possível notar uma diferença muito contrastante entre elas. A rede na cidade de Indaiatuba é menor do que aquela da cidade de Campinas, porém a de Indaiatuba possui mais fluxos entre seus componentes do que aqueles que pertencem a rede na cidade de Campinas. Nosso entendimento desse fato se baseou nas observações de campo e no acompanhamento realizado. As empresas na rede de Indaiatuba são muito próximas fisicamente umas das outras, algumas empresas estão no mesmo quarteirão ou apenas a alguns minutos de caminhada. Essa proximidade facilita os fluxos de informação de uma maneira mais pessoal e menos burocrática, criando assim o que denominamos de cumplicidade entre os gestores. $\mathrm{O}$ resultado foi um maior relacionamento entre esses atores.

REMark - Revista Brasileira de Marketing, São Paulo, v. 9, n. 2, p 166-189, mai./ago. 2010. 
Figura 3 - Resumo dos achados aplicados ao modelo teórico sobre a vantagem competitiva a partir dos relacionamentos da empresa.

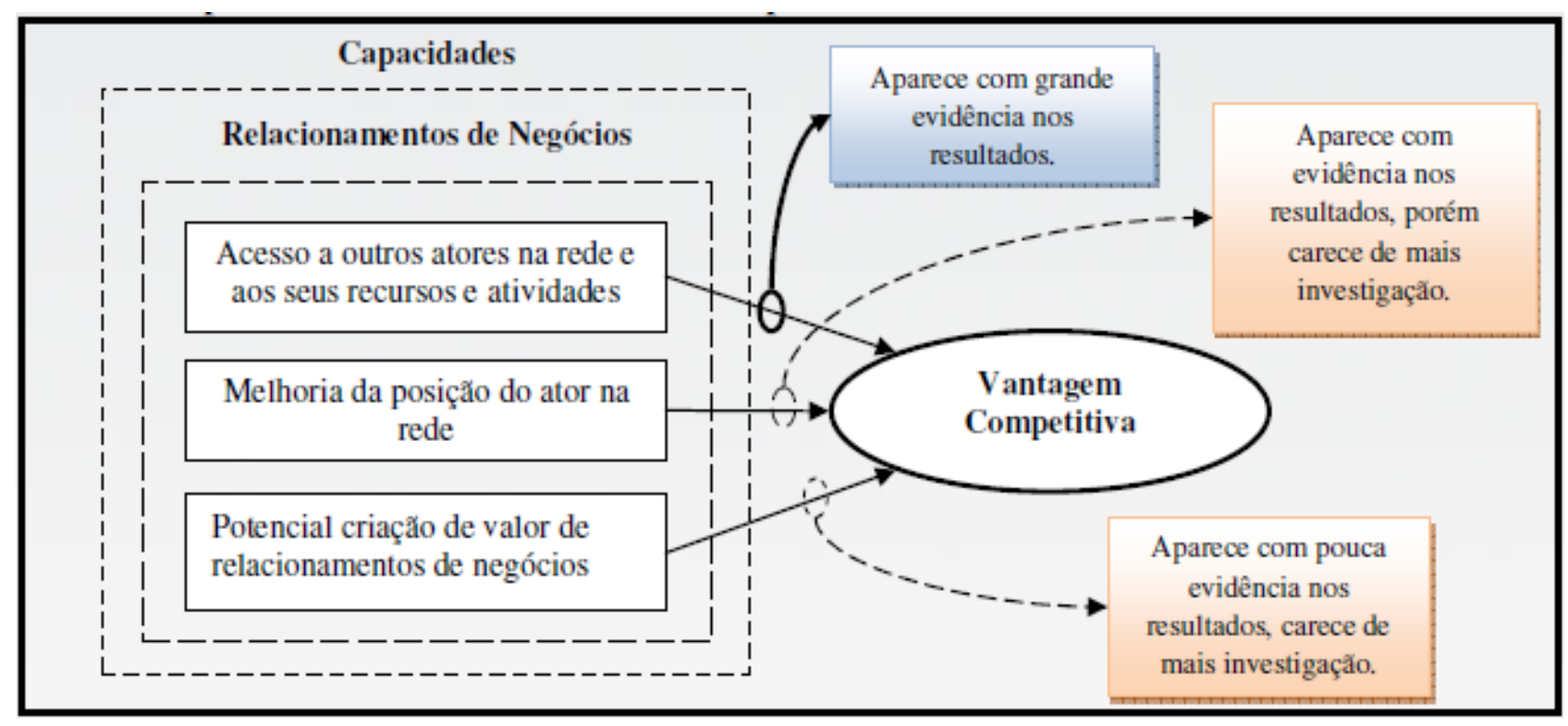

Fonte: Adaptado pelos autores de Alves e Tiergarten (2008).

Já a rede de Campinas possui outra configuração, a cidade é muito maior e as empresas se encontram espalhadas pela cidade. Apesar da facilidade dos meios de telecomunicações e das reuniões e encontros frequentes, é possível notar que estes ocorrem com uma assiduidade muito menor do que aqueles verificados em Indaiatuba.

Como limitação do estudo, pode-se colocar que a maior dificuldade encontrada foi o de desenvolver instrumentos de pesquisa de redes. Os pesquisadores utilizam predominantemente instrumentos tradicionais, como questionários, que buscam relações causais restritas. Mas quando se deseja entender os fenômenos de aspectos sociais, sendo este o foco do trabalho, esses instrumentos de pesquisa não auxiliam muito, pois acabam em variáveis estruturais métricas, tais como densidade e concentração, o que não resolvia nosso problema.

Também como limite metodológico deve ser citado o fato de a pesquisa ser de cunho qualitativo. Os resultados têm pouco campo de generalização se comparados a pesquisas quantitativas. Pesquisas qualitativas possibilitam a criação de hipóteses que geram novas pesquisas, permitindo que o pesquisador faça ligações com fenômenos paralelos ao objeto de estudo, como experiências pessoais.

REMark - Revista Brasileira de Marketing, São Paulo, v. 9, n. 2, p 166-189, mai./ago. 2010. 
Este estudo contribui para a discussão do tema redes de empresas. A maior implicação prática que pode advir desta pesquisa é levar aos gestores atuantes em empresas que estão participando de uma rede a importância estratégica que os relacionamentos possuem. Os gestores deverão considerar, dentro da formulação das suas estratégias, o desenvolvimento e a manutenção de relacionamentos para: terem acesso aos parceiros da rede e aos recursos que eles possuem e construírem uma posição forte dentro da rede de forma a poder influenciar outros parceiros.

Os pesquisadores apontam também a importância que a potencial criação de valor tem para a estratégia de uma empresa que decide se associar a uma rede de empresas. Toda empresa deverá, obrigatoriamente, focar seus esforços para criar valor através dos relacionamentos entre empresas; dessa forma, poderá ter melhores condições para acessar os parceiros de negócios e principalmente os recursos que eles poderão disponibilizar dentro da rede de empresas.

\subsection{RECOMENDAÇÕES PARA FUTUROS ESTUDOS}

Em face dessas constatações, entende-se como relevante a aplicação de novas pesquisas que possibilitem a ampliação da compreensão do tema, utilizando este estudo e outras metodologias como referência, de modo que os pesquisadores possam oferecer resultados que possam ser generalizados ao segmento. Nesse sentido, são recomendadas algumas ações que visem: Investigar PMEs de outros segmentos. O estudo de outros segmentos possibilitará a ampliação do entendimento da função dos relacionamentos de negócios e dos relacionamentos sociais.

Realizar um estudo, de forma mais aprofundada, da relação de confiança na formação de redes de PMEs. Uma investigação que considere apenas a relação da confiança na formação da rede seria importante para entender como esse mecanismo influencia a formação de uma rede.

Realizar um estudo descritivo, que investigue mais profundamente o constructo, melhoria da posição dentro da rede, do ponto de vista de governança e do poder, dentro das redes de empresas. Realizar um estudo quantitativo, relacionando a confiança e a quantidade de negócios, em números ou em volumes, entre os atores da rede. Esse tipo de estudo seria interessante e importante para conseguirmos dimensionar e relacionar os relacionamentos sociais e de negócios.

Investigar qual o papel dos relacionamentos antecedentes na formação de uma rede de PMEs. Um estudo histórico seria interessante para entender o que acontece antes da rede se formar.

Aqui é importante salientar a dificuldade de operacionalizar esse tipo de estudo, uma vez que o pesquisador precisaria estar presente no momento do nascimento de uma rede para que pudesse capturar as variáveis sociais, bem como as variáveis de negócios.

REMark - Revista Brasileira de Marketing, São Paulo, v. 9, n. 2, p 166-189, mai./ago. 2010. 
Fazer um estudo de casos múltiplos em redes de pequenas empresas utilizando-se técnicas quantitativas. As técnicas quantitativas permitem criar generalizações e possivelmente ajudarão na obtenção de respostas às hipóteses que discutam as interfaces entre as variáveis sociais e as variáveis de negócios.

Portanto, um estudo que envolva um maior número de empresas e que possa atender à generalização seria a principal recomendação deste trabalho.

\section{REFERÊNCIAS}

Alves, C. A., \& Tiergarten, M. (2008). A importância estratégica dos relacionamentos de negócios: uma visão baseada em competências da firma [CD-ROM]. In Anais do Encontro da Associação Nacional de Pós-Graduação e Pesquisa em Administração, 32. Rio de Janeiro: ANPAD.

Amit, R., \& Schoemaker, P. (1993). Strategic assets and organizational rent, Strategic Management Journal, 14(1), 33-46.

Bardin, L. (1977). Análise de conteúdo. Lisboa: Edições 70.

Barney, J. (1995). Looking inside for competitive advantage. Academy of Management Review, 9(4), 49-61.

Barney, J. (1991). Firm resources and sustained competitive advantage. Journal of Management, $17(1), 99-120$.

Barney, J., Wright, M., \& Ketchen, D. J., Jr. (2001). The resource based view of the firm: ten years after 1991. Journal of Management, 27(6), 625-641.

Collis, D. J. (1994). Research note: how valuable are organizational capabilities? Strategic Management Journal, 15(8), 143-152.

Conner, K. (1991). A historical comparison of resource based view and five schools of thought within industrial organization economics: do we have a new theory of the firm. Journal of Management, 7(1), 121-154.

REMark - Revista Brasileira de Marketing, São Paulo, v. 9, n. 2, p 166-189, mai./ago. 2010. 
Duyster, G., \& Lemmens, C. (2003). Alliance group formation: enabling and constraining effects of embeddedness and social capital in strategic technology alliance networks. International Studies of Management and Organization, 33(2), 49-68.

Dyer, J. H., \& Singh, H. (1998). The relational view: cooperative strategy and sources of interorganizational competitive advantage. Academy of Management Review, 23(4), 660-679.

Eisenhardt, K. M. (1989). Building theory from case study research. Academy of Management Review, 14(4), 532-550.

Fahy, J., \& Smithee, A. (1999). Strategic marketing and the resource based view of the firm. Academy of Marketing Science Review, 10, 1-19.

Fleury, A. C. C., \& Fleury, M. T. L. (2003). Estratégias competitivas e competências essenciais: perspectivas para a internacionalização da indústria no Brasil. Gestão e Produção, 110(2), 129144.

Gadde, L. E., Huemer, L., \& Hakansson, H. (2003). Strategizing in industrial networks. Industrial Marketing Management, 32(5), 357-64.

Gil, A. C. (2002). Como elaborar projetos de pesquisa (4a ed.). São Paulo: Atlas.

Grant, R. M. (1991). The resource-based theory of competitive advantage: implications of strategic formulation. California Management Review, 33(3), 114-134.

Gulati, R. (1998). Alliances and networks. Strategic Management Journal, 19(4), 293-317.

Gulati, R. (1995). Social structure and alliance formation patterns: a longitudinal analysis. Administrative Science Quarterly, 40(4), 619-652.

Gulati, R., Nohria, N., \& Zaheer, A. (2000). Strategic networks. Strategic Management Journal, 21(3), 203-15.

Herremans, I. M., \& Isaac, R. G. (2004). The intellectual capital realization process (ICRP): na application of the resource based view of the firm. Journal of Managerial Issues, 16(2), 217231.

Kogut, B., \& Zander, U. (1993). Knowledge of the firm and the evolutionary theory of the multinational corporation. Journal of International Business Studies, 29(3), 625-645.

REMark - Revista Brasileira de Marketing, São Paulo, v. 9, n. 2, p 166-189, mai./ago. 2010. 
Kraatz, M. S., \& Zajac, E. J. (2001). How organizational resources affect strategic change and performance in turbulent environments: theory and evidence. Organization Science, 12(5), 632657.

Lado, A. A., Boyd, N., Wright, P., \& Kroll, M. (2006). Paradox and theorizing within the resource based view. Academy of Management Review, 31(1), 115-131.

Lavie, D. (2006). The competitive advantage of interconnected firms: an extension of the resourcebased view. Academy of Management Review, 31(3), 638-658.

Leask, G. (2004). Strategic groups and the resource based view: natural complements enhancing our understanding of the competitive process. Birmingham: Aston Business School.

Lee, C., Lee, K., \& Pennings, J. M. (2001). Internal capabilities, external networks, and performance: a study on technology-based ventures. Strategic Management Journal, 22, 615640.

Loasby, B. (1998). The organization of capabilities. Journal of Economic Behavior and Organization, 35(2), 139-160.

Ludke, M., \& André, M. A. (1996). Pesquisa em educação: abordagens qualitativas. São Paulo: EPU.

Mattar, F. N. (1996). Pesquisa de marketing: edição compacta. São Paulo: Atlas.

Martins, G. A. (2006). Estudo de caso: uma estratégia de pesquisa. São Paulo: Atlas.

McEvily, B., \& ZAHEER, A. (1999). Bridging ties: a source of firm heterogeneity in competitive capabilities. Strategic Management Journal, 20(12), 1133-1156.

Morgan, R. M., \& Hunt, S. D. (1994). The commitment-trust theory of relationship marketing. Journal of Marketing, 58(3), 20-38.

Nohria, N., \& Garcia-Pont, C. (1991). Global strategic alliances and industry structure. Strategic Management Journal, 12(8), 105-124.

Patton, M. Q. (2002). Qualitative research and evaluation methods. Thousands Oaks: Sage.

Pfeffer, J., \& Nowak, P. (1976). Joint ventures and interorganizational interdependence. Administrative Science Quarterly, 21(3), 398-418.

REMark - Revista Brasileira de Marketing, São Paulo, v. 9, n. 2, p 166-189, mai./ago. 2010. 
Priem, R. L., \& Butler, J. E. (2001). Is the resource-based "view" a useful perspective for strategic management research? Academy of Management Review, 26(1), 22-40.

Saxton, T. (1997). The effects of partner and relationship characteristics on alliance outcomes. Academy of Management Journal, 40(2), 443-461.

Stuart, T. E. (2000). Interorganizational alliances and the performance of firms: a study of growth and innovation rates in a high-technology industry. Strategic Management Journal, 21(8), 719 811.

Stuart, T. E., Hoang, H., \& Hybels, R. (1999). Interorganizational endorsements and the performance of entrepreneurial ventures. Administrative Science Quarterly, 44(2), 315-349.

Teece, D. J., Pisano, G., \& Shuen, A. (1997). Dynamic capabilities and strategic management. Strategic Management Journal, 18(7), 509-533.

Vergara, S. C. (2003). Projetos e relatórios de pesquisa em administração. São Paulo: Atlas.

Vieira, M. M. F., \& Zouain, D. M. (2004). Pesquisa qualitativa em administração. Rio de Janeiro: FGV.

Wernerfelt, B. (1984). A resource-based view of the firm. Strategic Management Journal, 5(2), 171-180.

REMark - Revista Brasileira de Marketing, São Paulo, v. 9, n. 2, p 166-189, mai./ago. 2010. 


\title{
THE STRATEGIC IMPORTANCE OF BUSINESS RELATIONSHIP IN ENTERPRISE NETWORKS: A RESOURCE BASED VIEW - RBV
}

\begin{abstract}
This study intends to discuss the importance of building a co-operative strategic relationship in business, since it became an important precedent of success in business. Researchers have demonstrated that partnership and alliances have an important role in establishing a competitive advantage based on a firm's resources. For the objective in this research, we commence with na investigation in two real-state networks of which we adopt as a methodological pillar, the qualitative typology of investigation, due to it providing the most adequate observations. Methods utilised for this investigation included: a semi-structured interview; bibliographic research and nonparticipant. It concludes to demonstrate that the relationship researched proves the importance of a well-meaning cooperative strategy for companies acting in network formats. As a direct consequence, the access to partners and their resources could be considered the most strategic object analyzed. As a methodological limit, the fact this research is qualitative must be underlined and noted. These findings have little room for generalization when compared with quantitative research. The biggest practical implication is to allow managers participating in a network to perceive the strategic importance of this relationship. The manager must consider, within the boundaries of their formulated strategies, the development and maintenance to: grant them access to partners and their resources and the ability to build a strong position within the network in order to influence other partners.
\end{abstract}

Keywords: Enterprise Networks; Business Relationship; Strategic Resources; RBV.

Data do recebimento do artigo: 04/08/2010

Data do aceite de publicação: 01/10/2010

REMark - Revista Brasileira de Marketing, São Paulo, v. 9, n. 2, p 166-189, mai./ago. 2010. 